\title{
Temperature and metallicity maps in cool core clusters of galaxies
}

\author{
Silvia Caffi, Fabio Gastaldello, Simona Ghizzardi and Silvano Molendi \\ IASF-CNR Sez. Milano
}

\section{Observation and data analysis}

We have analized XMM-Newton data of A2199, a nearby $(z=0.030)$, relaxed, rich cluster of galaxies, whose X-ray emission is strongly peaked on the central galaxy, NGC6166, suggesting the existence of a cluster cool core (Peres et al. 1998).

The background subtraction is performed using an observation characterized by features similar to those of the A2199 observation. We have selected, in the XMM-Newton archive, a field observation presenting the following properites:

- duration of about $90 \mathrm{ksec}$;

- performed with the same filter as the A2199 observation;

- characterized by a galactic HI column density and a soft X-ray background, the latter being calculated from the ROSAT All-Sky survey diffuse background maps, which differ from those of the A2199 observation $\left(N_{H}=8.7 \times 10^{19} \mathrm{~cm}^{-2}, R_{45}=208.2 \times\right.$ $10^{-6} \mathrm{cts} / \operatorname{arcmin}^{2}$ ) by $3 \%$ and $30 \%$ respectively.

We have accumulated spectra from 9 concentric annuli, out to 12 arcmin, centered on the X-ray emission peak and have fit them with a single temperature model (mekal * wabs model in XSPEC) in the $1.5-10.0 \mathrm{keV}$ spectral band, well suited for temperature measurements in the range $k T \sim[3,5] \mathrm{keV}$.

We have then deprojected the radial profiles using the technique described in Ettori et al. (2002).

\section{Results}

\subsection{Projected profiles}

Here we present the projected profiles of temperature and iron abundance (Fig. 1) plotted together with Chandra and BeppoSAX profiles derived from Johnstone et al. (2002) and De Grandi \& Molendi (2001, 2002).

While the temperature profiles derived from XMM-Newton and Chandra data show a remarkable agreement, the comparison between BeppoSAX and our data points toward a systematic difference of about $10 \%$, already noted in the Coma cluster: $0.8 \mathrm{keV}$ on a temperature of $8.2 \mathrm{keV}$ (De Grandi \& Molendi, 2002).

There is a clear difference in the abundance profiles of XMM-Newton and Chandra. This difference could be due to the proximity of the $F e-K_{\alpha}$ line at $6.7 \mathrm{keV}$, used to mesure the metallicity, to the spectral upper limit in Johnstone et al.'s analysis $(7.0 \mathrm{keV})$.

\subsection{Deprojected profiles}

We have deprojected radial profiles using the method indicated by Ettori et al. (2002), obtaining three-dimensional profiles for temperature, abundance (see Fig. 2), electron density $\left(n_{e}\right)$, emissivity $(\epsilon)$, cooling time $\left(t_{c o o l}\right)$, pressure (see Fig. 3 left panel), entropy, gravitational mass, iron mass and iron mass excess (see Fig. 3 right panel). 

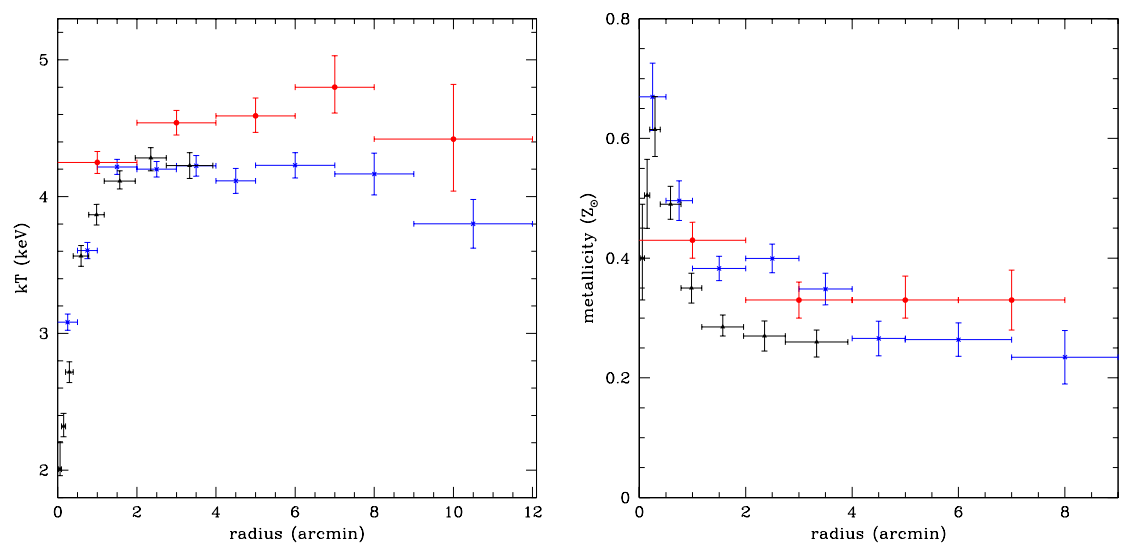

Figure 1. Temperature (left) and iron abundance (right) projected profiles: blue, red and black points are, respectively, for XMM-Newton, BeppoSAX and Chandra data.

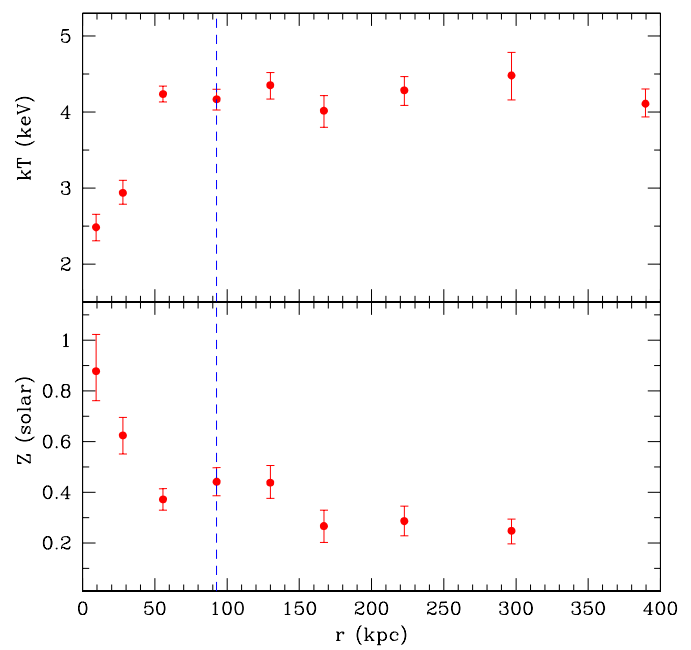

Figure 2. Temperature and iron abundance deprojected profiles.

The radius within which the temperature (abundance) profile decreases (increases) is about a factor of 2 smaller than the cooling radius, estimated from the $t_{\text {cool }}$ profile. The value of $r_{c o o l}$ is about $95 \mathrm{kpc}$, in good agreement with previous estimates by Perez et al. (1998) and Johnstone et al. (2002).

The total iron mass excess is $6.3 \times 10^{8} \mathrm{M}_{\odot}$, a factor of 2 smaller than previously estimated in De Grandi et al. (2003) with BeppoSAX data. The discrepancy could be due to the differences between the projected metallicity profiles (see Fig. 1).

\section{References}

Peres C. B. et al. 1998 MNRAS 298416

De Grandi S. \& Molendi S. 2001 ApJ 551153

De Grandi S. \& Molendi S. 2002 ApJ 567163

De Grandi S. et al. 2003 astro-ph/0310828, A\&A, in press 

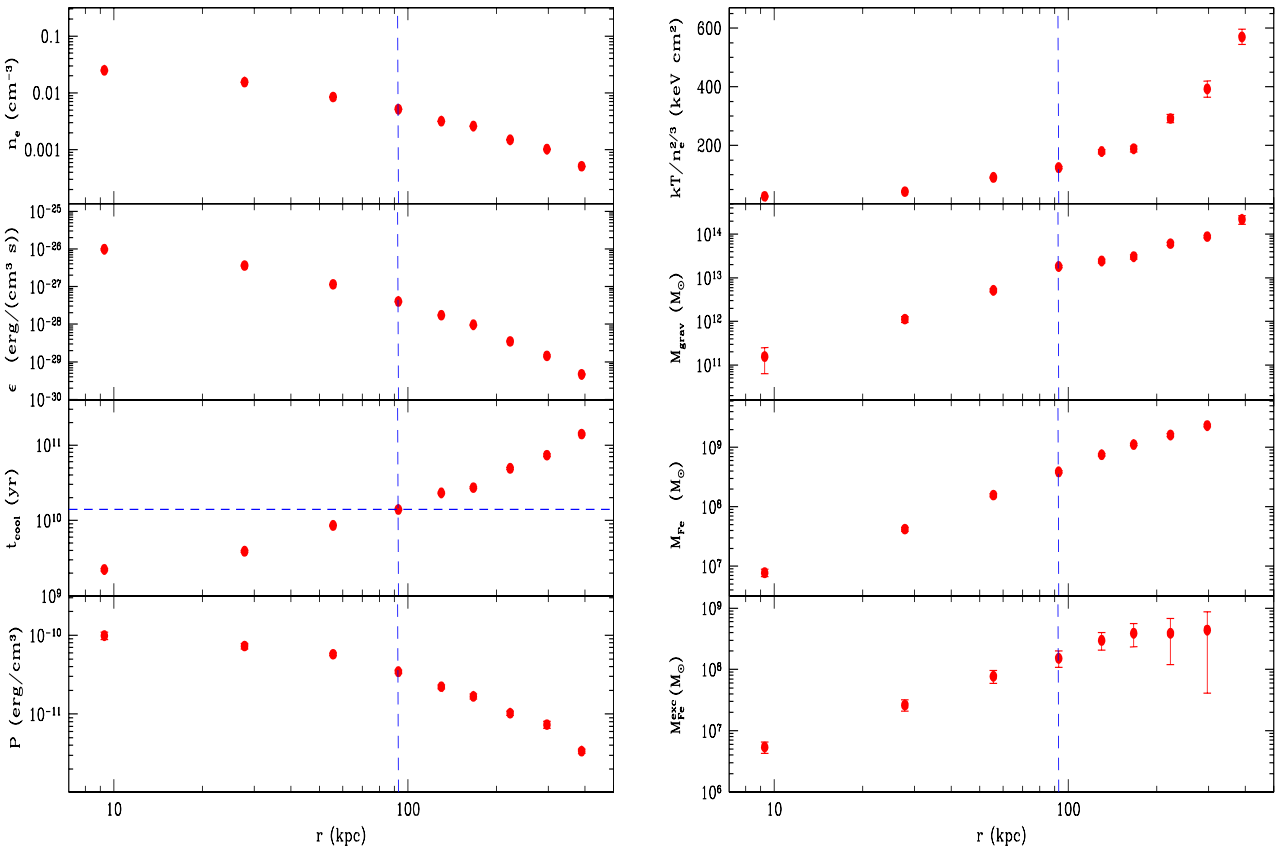

Figure 3. Deprojectd quantities of A2199: $n_{e}, \epsilon, t_{c o o l}, P$ in the left panel, entropy (defined as $\left.k T / n_{e}^{2 / 3}\right), M_{g r a v}, M_{F e}$ and $M_{F e}^{e x c}$ in the right panel.

Ettori S. et al. 2002 A\&A 391841

Johnstone R. M. et al. 2002 MNRAS 336299 